\title{
Distribution of Filipin-Sterol Complexes in Japanese Abalone Sperm During the Acrosome Reaction
}

\author{
Yoko Shiroya and Yoshi T. Sakai \\ Biological Laboratory, Wayo Women's University, Ichikawa, Chiba 272, \\ Japan
}

\begin{abstract}
The distribution of membrane sterols in the acrosome reaction of Japanese abalone sperm was studied by freeze-fracture techniques using a polyene antibiotic, filipin, as a probe for membrane sterols. In the unreacted sperm, the plasma membrane covering the acrosome showed a random distribution of filipin-sterol complexes, while in the outer acrosomal membrane, filipinsterol complexes were rarely observed. In the initial stage of the acrosome reaction, filipin-sterol complexes were found densely distributed in the plasma membrane throughout the acrosomal region. On the subjacent outer acrosomal membrane, filipin-sterol complexes were densely packed in the apical area, corresponding to the truncated cone region. This apical area consistently showed such regionalization of filipin-sterol complexes during the acrosome reaction. As the acrosomal process elongated, the intramembrane particle (IMP)-free areas which appeared in the acrosomal process membrane were found to be displaced by densely distributed filipin-sterol complexes. These results indicate a possible regionalization of membrane sterols related to the acrosome reaction.
\end{abstract}

In marine invertebrates the acrosome reaction consists fundamentally of two requisites; exocytosis of the acrosomal vesicle to release lytic substances for dissolving the egg coats, and exposure of the newly formed acrosomal process to generate a site for fusion with the egg. Of particular interest is that membrane transformations occurring during the acrosome reaction involve the fusion between the plasma membrane and the outer acrosomal membrane at the apex of the acrosome, resulting in exocytosis, and rapid extension of the inner acrosomal membrane to form the acrosomal process membrane which may eventually fuse with the egg plasma membrane.

A few freeze-fracture studies of abalone (29) and mammalian sperm (9, 10, 15, 40) have demonstrated the regionalization of intramembrane particles (IMPs) associated with the acrosome reaction, while little information is available concerning the distribution of sterols in the sperm membrane during the acrosome reaction (12). Sterol is an important component of the cell membrane and plays a fundamental role in modulating the permeability and fluidity of membranes $(6,24,33)$. Thus, the study of the distribution of sterol in the membranes is important in order to further understand the structural properties and functions of the membrane in relation to the acrosome reaction.

In the present study, attempts have been made to investigate the distribution of sterols (usually cholesterol) in the membranes of abalone sperm during the 
acrosome reaction using a polyene antibiotic, filipin. Filipin has been shown to interact specifically with membrane cholesterols and the related 3-hydroxysterols $(2,3,4,22)$, producing unique hemispherical lesions which can be recognized in freeze-fracture replicas and in thin sections $(5,7,18,25,27,37,39)$. A preliminary report of this work has been presented (35).

\section{MATERIALS AND METHODS}

Mature shells of Haliotis discus were provided by the Chiba Prefectural Fisheries Experimental Station, Chikura, Japan. Sperm shedding was induced by exposing the mature animals to ultraviolet (UV)-irradiated sea water $(16,17)$ and the sperm were then collected (29). The acrosome reaction was induced by ionophore A 23187, as described previously (36).

Filipin (Sigma) was initially dissolved in dimethyl sulfoxide and added to a fixative solution containing $2.5 \%$ glutaraldehyde and $1 \%$ paraformaldehyde in a $0.1 \mathrm{M}$ cacodylate buffer, $\mathrm{pH}$ 7.4. The cells were either incubated for $2 \mathrm{~h}$ in the dark at room temperature in the fixative solution containing $200 \mu \mathrm{g} / \mathrm{ml}$ filipin, or prefixed in the fixative solution for $30 \mathrm{~min}$ and then incubated in the presence of filipin, as described above. Control samples were fixed without filipin. After filipin treatment, the cells were washed with a $0.1 \mathrm{M}$ cacodylate buffer, $\mathrm{pH}$ 7.4.

Freeze-fracture samples were incubated in a $30 \%$ glycerol solution, placed in wells on a copper plate and then rapidly frozen in liquid Freon 22 cooled with liquid nitrogen. The samples were fractured and replicated in a JFD-7000-apparatus (JEOL) at $10^{-6}$ Torr at $-115^{\circ} \mathrm{C}$. The fractured specimens were shadowed with platinum and coated with carbon. The replicas were cleaned with sodium hypochlorite for $1 \mathrm{~h}$, washed with distilled water and mounted on carbon-coated 200 mesh copper grids.

For thin sections, the filipin-treated samples were postfixed in $1 \%$ osmium tetroxide, dehydrated in graded ethanol and embedded in Epon 812. The thin sections were stained with uranyl acetate and lead citrate. All replicas and thin sections were examined with a Hitachi HS-9 electron microscope.

\section{RESULTS}

Examination of the freeze-fracture images of abalone sperm treated with filipin revealed the presence of filipin-sterol complexes, which appeared as either hemispherical protuberances or pits in the membrane. The protuberances were about $30 \mathrm{~nm}$ in diameter on the $P$ (protoplasmic) face, whereas on the $E$ (extracellular) face the complexes appeared mainly as slightly smaller pits. Slightly rugged features were also often observed on the fracture-face.

Filipin treatment during fixation or following fixation showed no difference in the distribution of filipin-sterol complexes. The following observations were determined.

Unreacted (intact) sperm:

In the unreacted sperm, filipin-sterol complexes were randomly distributed in the density of $75-100$ per $\mu \mathrm{m}^{2}$ throughout the $\mathrm{P}$ and $\mathrm{E}$ faces of the plasma membrane covering the acrosome (Fig. 1). In contrast to the plasma membrane, the outer acrosomal membrane was nearly devoid of filipin-sterol complexes in both the $\mathrm{P}$ and $\mathrm{E}$ faces (Fig. 2). In this outer acrosomal membrane, numerous IMPs were randomly distributed throughout the $P$ face, while a few IMPs were scattered on the $E$ face. These IMP distributions coincide with our previous freeze-fracture observations in sperm that have not been treated with filipin (29). 

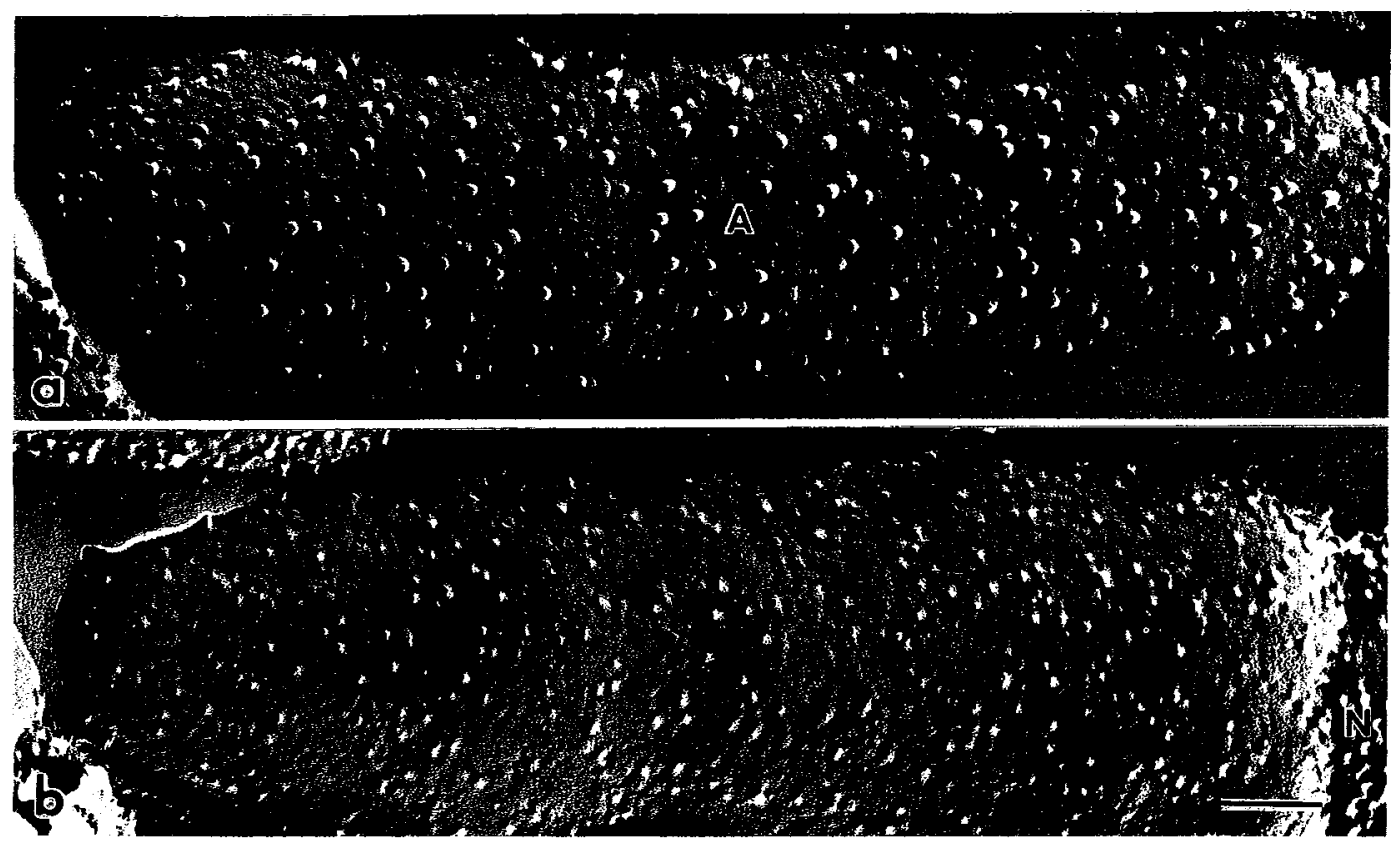

Fig. 1. Freeze-fracture replicas of the plasma membranes of unreacted abalone sperm treated with filipin. Filipin-sterol complexes are homogeneously scattered on both the $P$ (a) and the $E$ (b) faces in the acrosomal region (A). In the nuclear area $(\mathrm{N})$, filipin-sterol complexes are densely packed. Bar= $0.25 \mu \mathrm{m}$.

Observations of thin sections of sperm treated with filipin provided additional information on ultrastructural changes of the membrane induced by filipin. In the filipin-treated sperm, the plasma membrane covering the acrosome displayed deformations induced by the formation of the filipin-sterol complex which appeared as tiny bubbles or bumps scattered on the plasma membrane (Fig. 3). However, in the control sample, which lacked filipin treatment, no such membrane deformations were observed. In the outer and inner acrosomal membranes, filipin-induced deformations were seldom observed in the filipin-treated sperm. These membrane deformations induced by filipin-sterol complexes, as observed in the thin sections, correlate well with the freeze-fracture images.

Acrosome-reacted sperm:

Abalone sperm were induced to undergo the acrosome reaction by the addition of ionophore A 23187 to sea water in the presence of $40 \mathrm{mM} \mathrm{CaCl}_{2}$. The process of the acrosome reaction focused on obvious membrane transformations involving exocytosis of the acrosomal vesicle, and rapid extension of the inner acrosomal membrane to form the acrosomal process membrane with simultaneous elongation of the acrosomal process. Freeze-fracture observations of the acrosome-reacted sperm treated with filipin revealed distinct variations in filipin labelling, corresponding to these membrane transformations.

At the initial stage of the acrosome reaction, just before vacuolation occurred in the trigger region, the plasma membrane covering the acrosome became heavily 


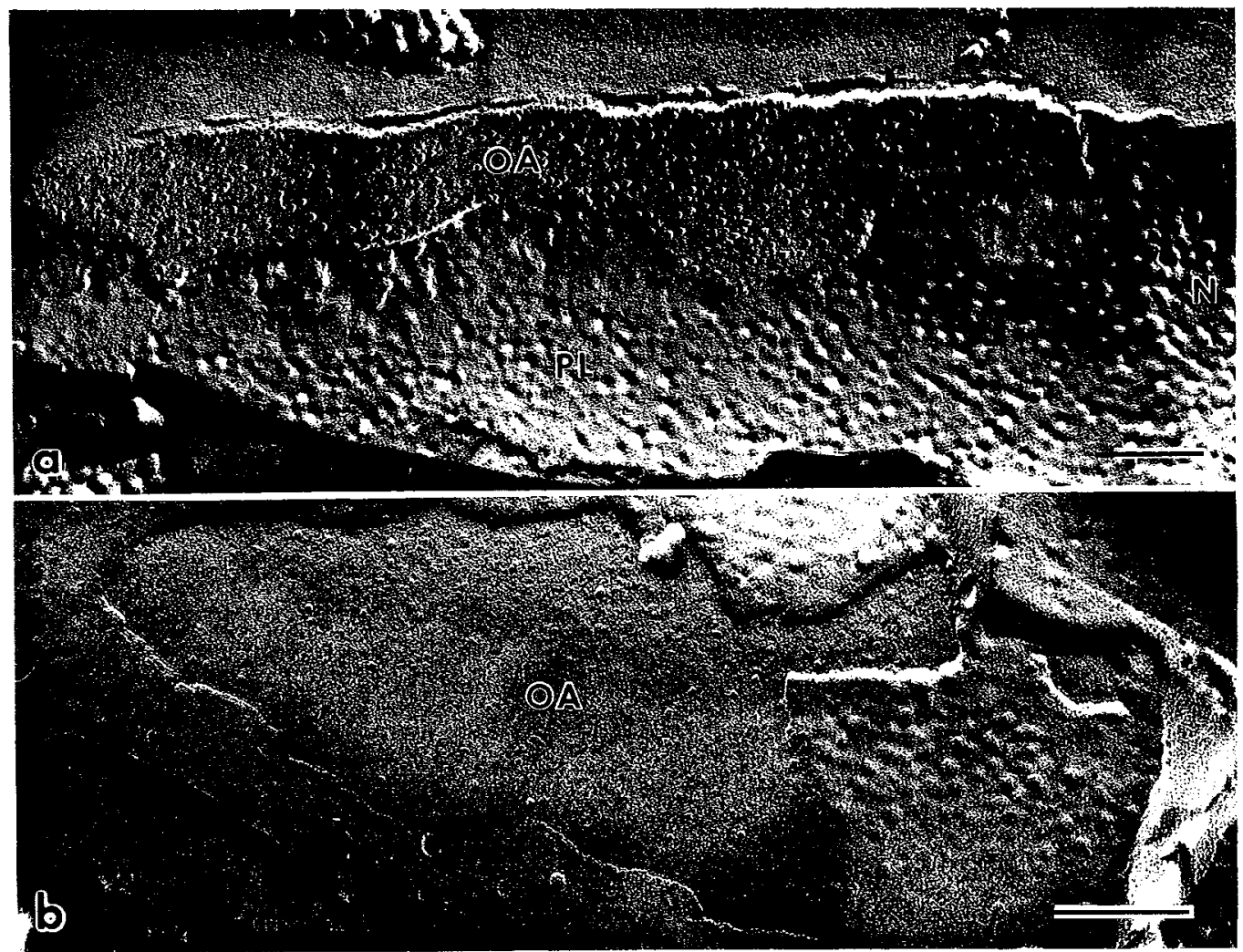

Fig. 2. Freeze-fractured outer acrosomal membranes of unreacted sperm after filipin treatment. Both the P (a) and the E faces (b) are nearly devoid of filipin-sterol complexes. Numerous IMPs are randomly distributed throughout the $\mathbf{P}$ face, while the $\mathbf{E}$ face displays only a few IMPs. N, nucleus; OA, outer acrosomal membrane; PL, plasma membrane. Bar $=0.25 \mu \mathrm{m}$.

labelled with filipin-sterol complexes in both the $\mathrm{P}$ and $\mathrm{E}$ faces, giving an overall knobby appearance (Fig. 4). This knobby feature usually appeared as projections in the $\mathbf{P}$ face and as complementary depressions in the $E$ face. The subjacent outer acrosomal membrane, however, exhibited sparse distribution of the filipin-sterol complexes and was liable to increase the density of the filipin-sterol complexes towards the anterior aspect of the acrosome (Fig. 5).

As opening at the acrosomal apex occurred, filipin-sterol complexes became more densely packed on the outer acrosomal membrane in the periphery of the opening. This caused the formation of a sharp demarcation in the density of filipin-sterol complex (Fig. 6) at the site where the posterior end of the truncated cone was connected to the outer acrosomal membrane (34). Thus, the apical area showed a dense distribution of filipin-sterol complexes, which corresponds to the region where the truncated cone is directly subjacent to the outer acrosomal membrane. The remainder of the outer acrosomal membrane showed sparse distribution of the filipin-sterol complexes (Fig. 6). Even when the acrosomal contents were completely released, causing the acrosome to become slender, the densely packed filipin-sterol 


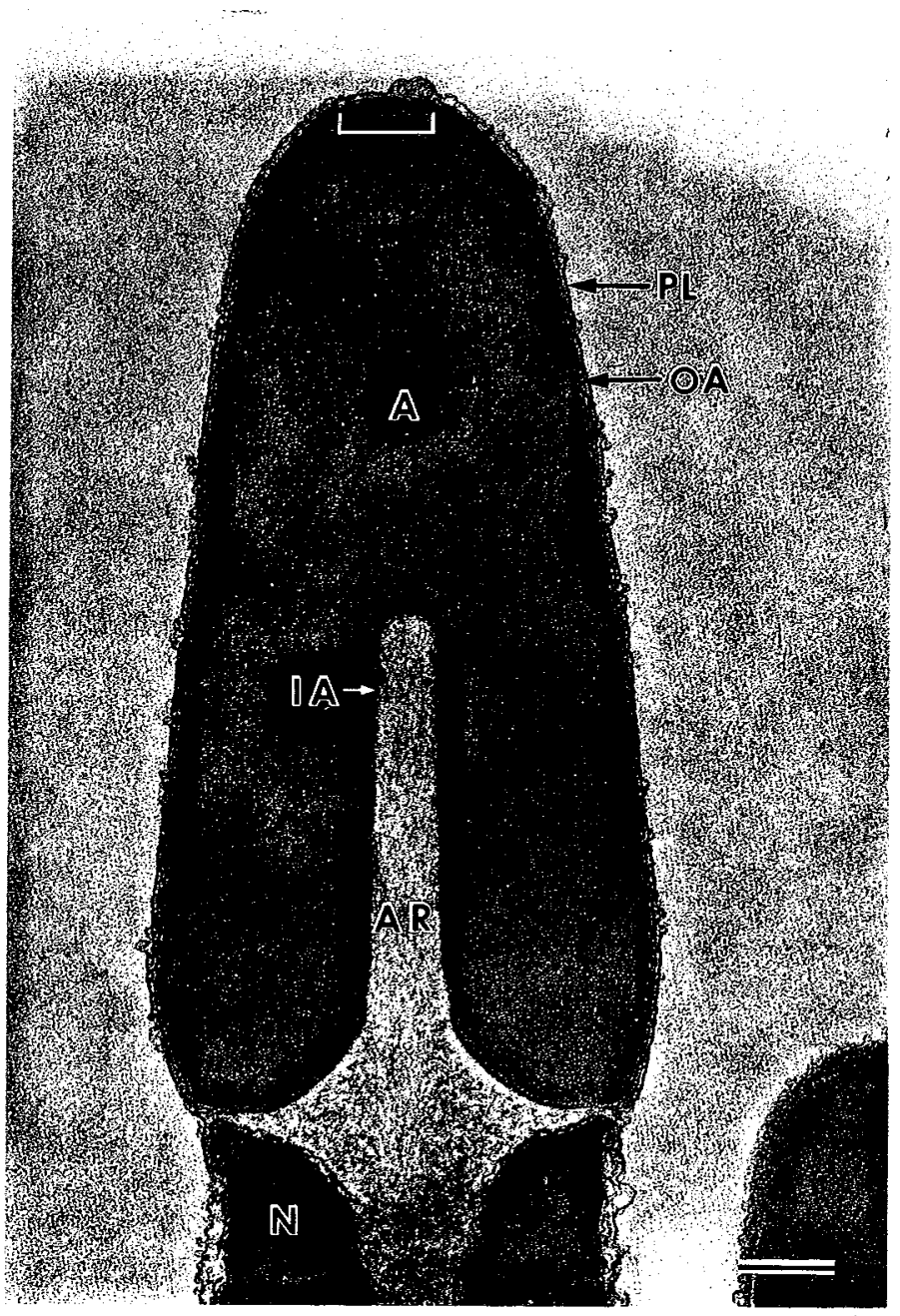

Fig. 3. Thin section through the acrosomal region of the unreacted abalone sperm treated with filipin. Filipin-sterol complexes are detected as tiny bubbles on the plasma membrane (PL) covering the acrosome (A). Membrane deformations induced by filipin are scarce in the outer (OA) and inner (IA) acrosomal membranes. Bracket indicates the trigger region. AR, axial rod; N, nucleus. Bar $=0.25 \mu \mathrm{m}$.

complexes were still clearly observed in the periphery of the acrosomal opening on both the $\mathrm{P}$ and $\mathrm{E}$ faces of the outer acrosomal membrane (Fig. 7). This distinct filipin labelling remained throughout the acrosome reaction.

As the axial rod elongated anteriorly to form the acrosomal process, the inner acrosomal membrane, which limits the anterior half of the axial rod, extended simultaneously and was transformed into the acrosomal process membrane covering the lengthening acrosomal process. In the initial stages of this membrane transformation, only a few filipin-sterol complexes were sparsely distributed in the funnel-shaped area at the posterior end of the acrosomal process membrane (Fig. 


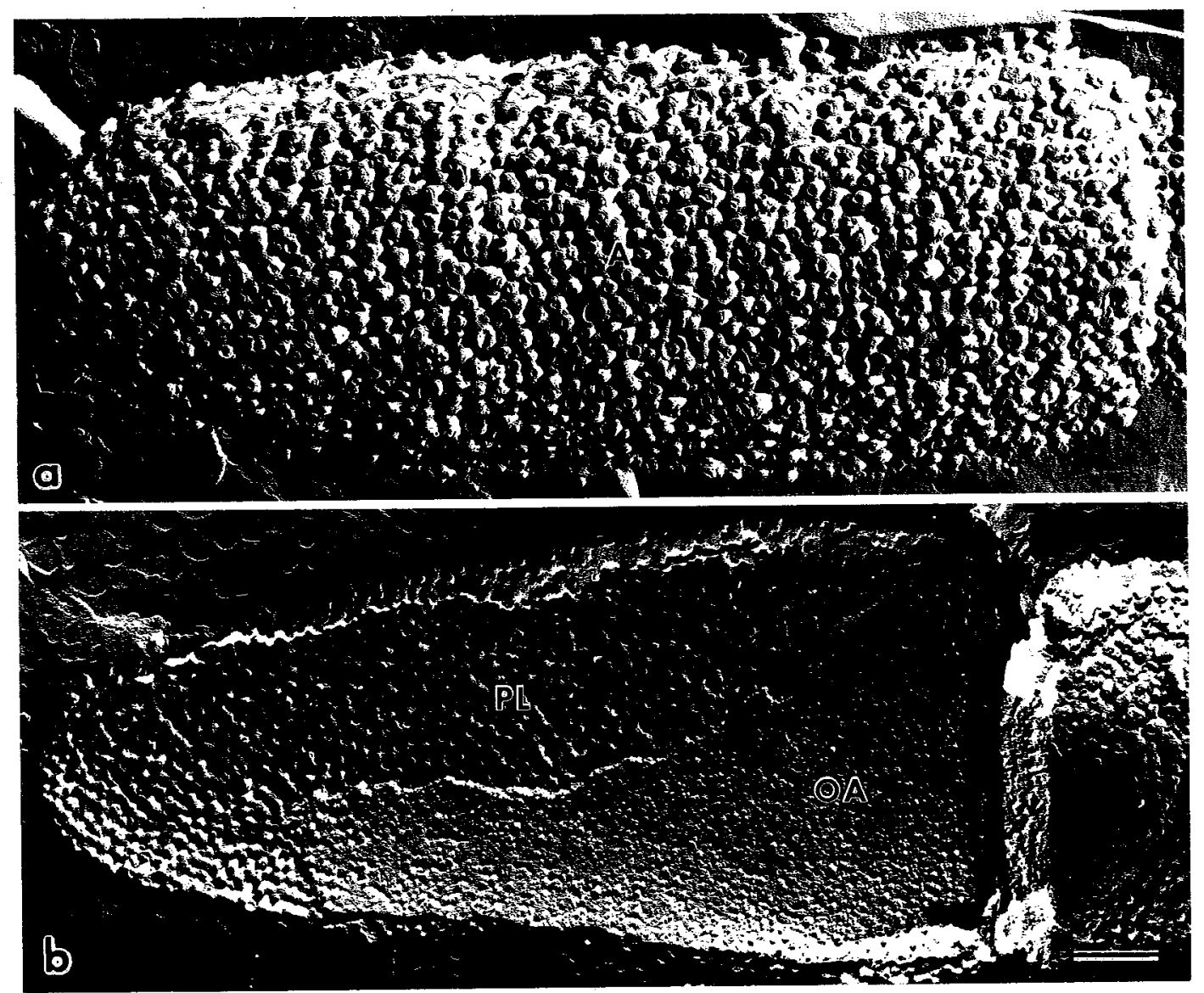

Fig. 4. Freeze-fracture replicas of the plasma membranes at the initial stage of the acrosome reaction, after filipin treatment. Filipin-sterol complexes are densely distributed on both the $\mathbf{P}(\mathrm{a})$ and the $\mathrm{E}$ (b) faces. The $E$ face of the outer acrosomal membrane (OA) with sparse distribution of filipin-sterol complexes is also indicated (b). A, acrosome; PL, plasma membrane. Bar $=0.25 \mu \mathrm{m}$.

8a) where numerous IMPs were densely packed. As the acrosome reaction proceeded, the density of the filipin-sterol complexes increased in this funnel-shaped area in the $\mathbf{P}$ face of the acrosomal process membrane, whereas the densely packed IMPs scattered sporadically leaving small groups of IMPs in a lattice-like pattern on the $P$ face (Fig. 8b). Thus, this IMP-free area appeared to be replaced with the more abundant filipin-sterol complexes. The shaft region of the acrosomal process membrane lengthening in the vacant acrosome initially showed numerous IMPs densely packed in a lattice-like pattern with a few filipin-sterol complexes scattered inbetween the IMPs (Fig. 9a). When the elongation of the acrosomal process proceeded further, this lattice-like arrangement of IMPs changed into a rhombic pattern accompanying IMP-free areas in patches along the acrosomal process. These IMP-free areas were then displaced by the dense arrangement of filipin-sterol complexes (Figs. 9b, c, d). In the region where the acrosomal process was protruding through the truncated 

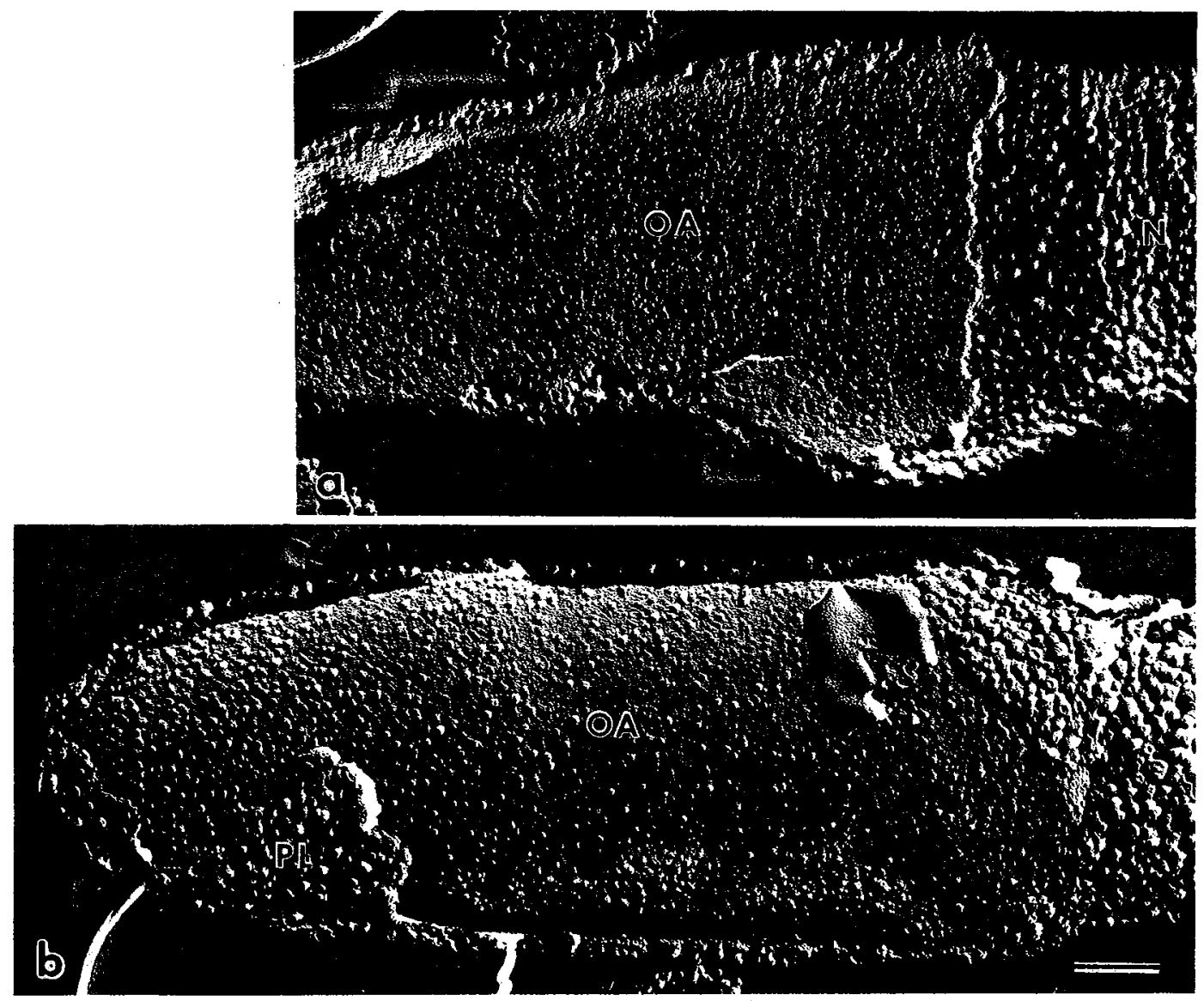

Fig. 5. Freeze-fracture replicas of the outer acrosomal membranes during the acrosome reaction. Both the P (a) and the $\mathrm{E}$ (b) faces display sparse distribution of filipin-sterol complexes throughout the acrosome just prior to the occurrence of the opening of the acrosomal apex. N, nucleus; PL, plasma membrane; $\mathrm{OA}$, outer acrosomal membrane. Bar $=0.25 \mu \mathrm{m}$.

cone, abundant filipin-sterol complexes were more widely distributed, while IMPs were scattered in the area devoid of the filipin-sterol complexes (Fig. 9e).

In thin sections, these membrane deformations induced by filipin could be clearly observed throughout the acrosomal process membrane. In the initial stage of the acrosome reaction, the plasma membrane became heavily labelled with filipin, causing it to have a wavy appearance. The shaft region of the inner acrosomal membrane showed wrinkles, whereas in the funnel-shaped region of the inner acrosomal membrane, no obvious deformations were observed (Fig. 10a). As the acrosomal process elongated, however, wavy configurations appeared in both the shaft and the funnel-shaped regions of the acrosomal process membrane. This indicated that the filipin-induced deformations were much heavier in the anterior region of the acrosomal process membrane (Fig. 10b). 

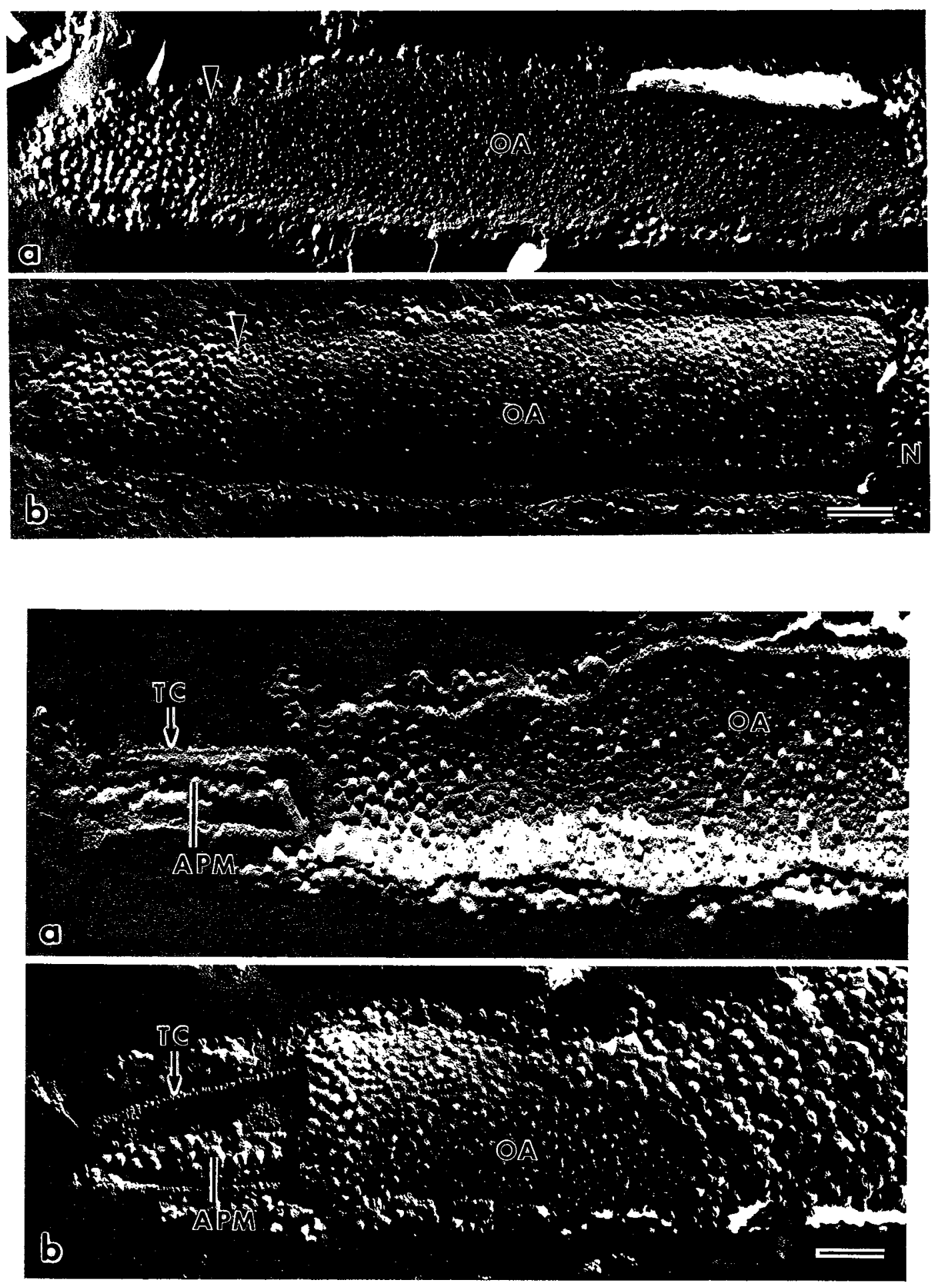

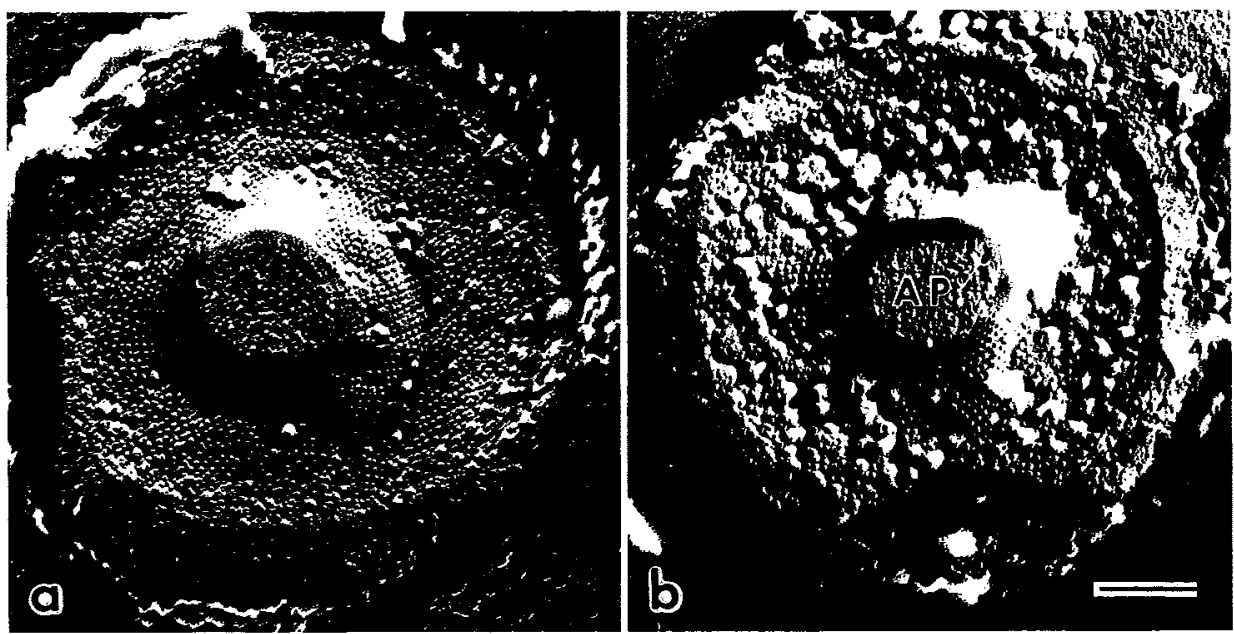

Fig. 8. The $\mathrm{P}$ face of the acrosomal process membrane labelled by filipin in the funnel-shaped region. (a) In the initial stages of the acrosome reaction, only a few filipin-sterol complexes are seen, whereas the IMPs are densely packed. (b) During the elongation stage of the acrosomal process (AP), the filipin-sterol complexes become densely distributed. Small groups of IMPs in a lattice-like pattern are scattered inbetween the filipin-sterol complexes. Bar $=0.2 \mu \mathrm{m}$.

\section{DISCUSSION}

Our present results using filipin as an ultrastructural probe for membrane sterols revealed distinct alterations in the distribution of sterols within the plasma and acrosomal membranes associated with the acrosome reaction. Though a few studies have already been performed in mammalian sperm on the membrane sterol distribution $(1,11,13,38)$, this study is the first to use invertebrate sperm in studies concerning sterol localization in membranes, including internal membranes such as the outer and inner acrosomal membranes, during the acrosome reaction.

The plasma membrane covering the acrosome in the unreacted (intact) sperm presents a homogeneous distribution of filipin-sterol complexes, whereas the outer and inner acrosomal membranes generally lack these complexes. This suggests a possible difference in free-sterol content or membrane reactivity toward filipin. Since no change in the distribution of the filipin-sterol complexes was found even in

Fig. 6. The outer acrosomal membrane in the initial stage of the acrosomal apex opening. Note that the filipin-sterol complexes are densely packed in the apical region of the acrosome, while the remaining area exhibits only sparse distribution of the filipin-sterol complexes on both the $P$ (a) and the $E$ (b) faces. Arrowheads denote the sharp demarcation in the density of filipin-sterol complexes which corresponds to the region where the truncated cone is connected to the outer acrosomal membrane (OA). N, nucleus. Bar $=0.25 \mu \mathrm{m}$.

Fig. 7. The acrosomal region of the outer acrosomal membrane at the stage of the elongation of the acrosomal process protruding out of the acrosomal apex. Note the filipin-sterol complexes densely packed in the periphery of the acrosomal opening on both the $P$ (a) and the $E$ (b) faces. The truncated cone (TC) is clearly observed projecting from the opening of the acrosome. The acrosomal process membrane (APM), densely labelled with filipin, is also indicated. OA, outer acrosomal membrane $\mathrm{Bar}=0.2 \mu \mathrm{m}$. 


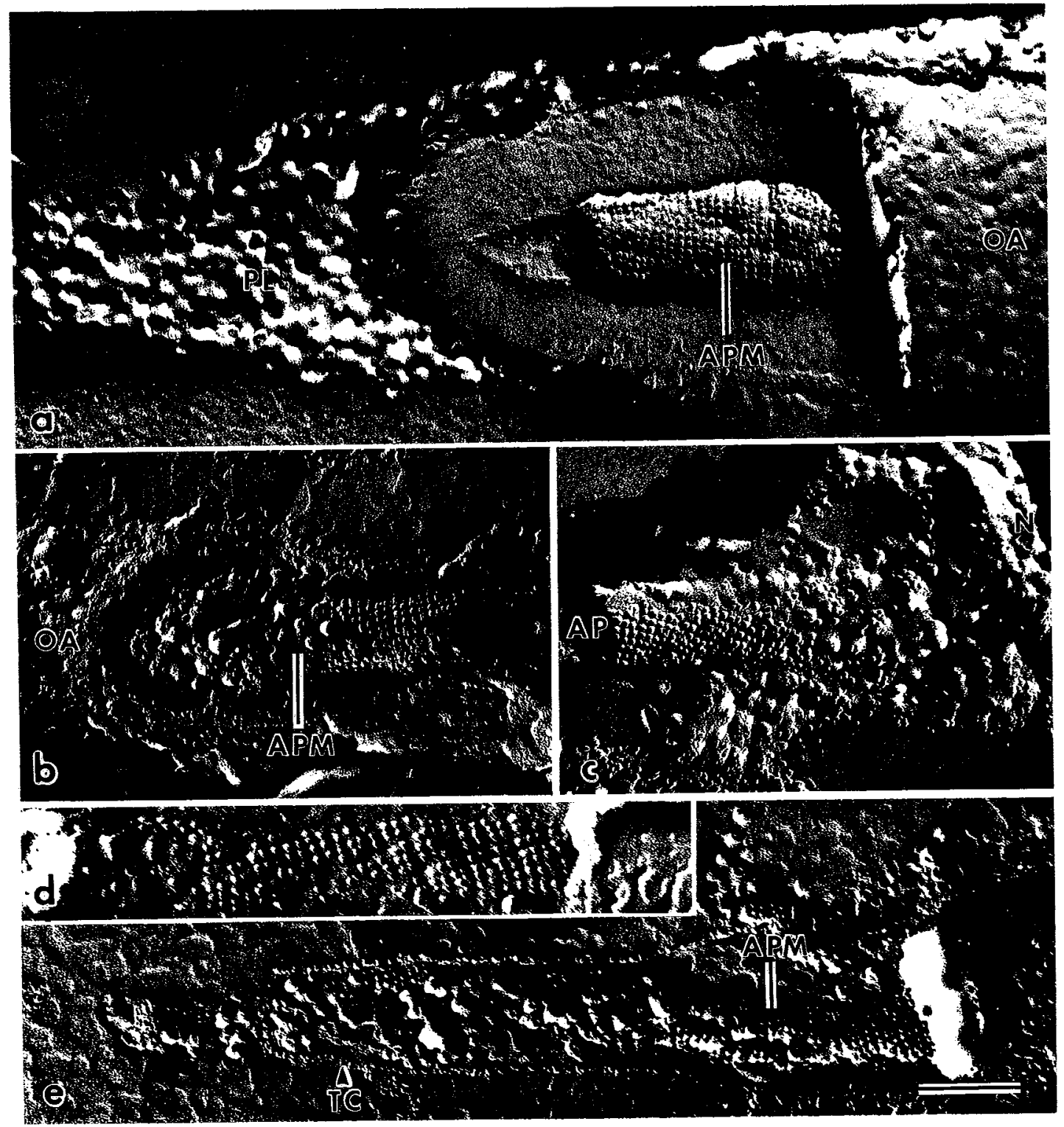

Fig. 9. The shaft region of the acrosomal process elongating during the acrosome reaction. (a) The acrosomal process beginning to elongate in the vacant acrosome. Note the IMPs densely packed in a lattice-like pattern and a few filipin-sterol complexes distributed between the packed IMPs. (b, c, d) The acrosomal process membrane (APM) elongating in the vacant acrosome. Note the filipin-sterol complexes distributed in the IMP-free areas in the anterior (b) and posterior (c) regions. The lattice-like pattern of the IMPs (a) changes into a rhombic pattern $(b, c)$ associated with the growth of the acrosomal process (AP). (d) A rhombic distribution of the IMPs is clearly seen in the further elongated acrosomal process. (e) The acrosomal process membrane (APM) further protruding through the cylinderized truncated cone (TC) shows heavy filipin labelling, while the IMPs distribute in areas lacking filipinsterol complexes. OA, outer acrosomal membrane; $N$, nucleus. Bar $=0.2 \mu \mathrm{m}$. 

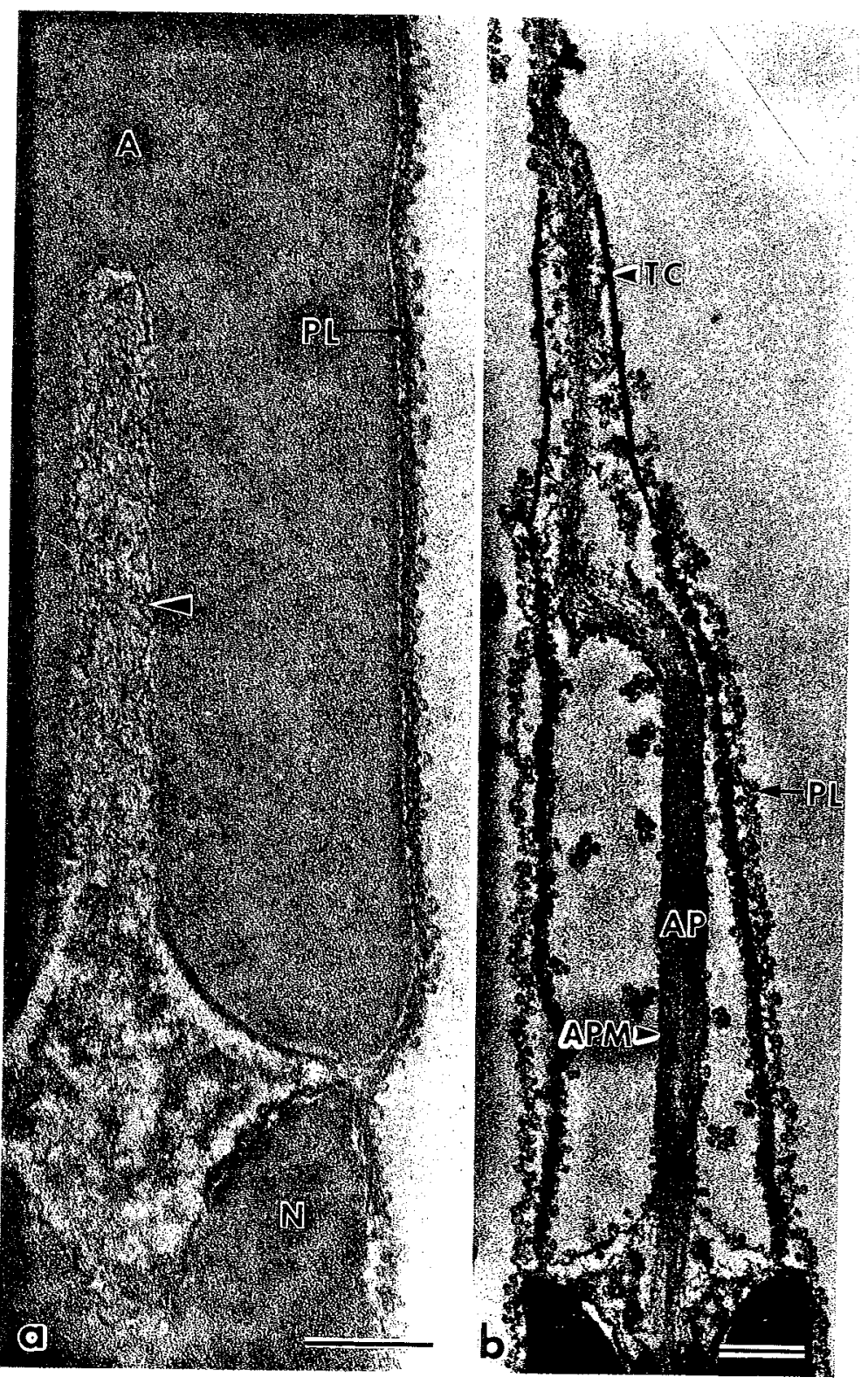

Fig. 10. Thin sections of the acrosome-reacted sperm after filipin treatment. (a) At the initial stage of the acrosome reaction, the plasma membrane (PL) covering the acrosomal region (A) is heavily labelled with filipin. The inner acrosomal membrane (arrowhead) is wrinkled in the shaft region, while no wrinkles are observed in the funnel-shaped region. (b) The acrosomal process (AP) elongates through the vacant acrosome protruding out of the truncated cone (TC). The plasma membrane (PL) shows a pronounced wavy appearance throughout the acrosomal region. The wavy or bubbled configuration is also observed in the shaft region as well as in the funnel-shaped region of the acrosomal process membrane (APM). N, nucleus. Bar $=0.25 \mu \mathrm{m}$. 
the sperm treated with filipin for $20 \mathrm{~h}$ (data not shown), this difference in the distribution of filipin-sterol complexes does not appear to be a consequence of poor diffusion of the probe. It is noteworthy that similar results have also been observed in the sperm of the guinea pig, mouse and hamster $(7,19,38)$, thus revealing little or no filipin-sterol complexes in the internal membranes. Such filipin-negative membranes in sperm cells, yet hitherto observed in limited species, might be related to a common feature in acrosomal membranes which enclose lytic substances.

It has been demonstrated that the density of filipin-sterol complexes decreases in regions where membrane fusion takes place, such as exocytosis in the mast cells, and myoblast fusion $(21,30,31)$. Our present results revealed that in the initial stage of the acrosome reaction, a significant increase in the density of the filipin-sterol complexes emerges in the plasma membrane covering the entire acrosomal region. Simultaneously, the outer acrosomal membrane exhibits heavy filipin labelling in the apical region of the acrosomal vesicle. These distinct dense distributions of filipin-sterol complexes may indicate a conspicuous increase in sterol distribution, which may in turn be an indication of the initiation of the acrosome reaction. Of particular interest is that these dense distributions of filipin-sterol complexes are maintained in both the plasma and outer acrosomal membranes, even after these membranes have become fused at the acrosomal apex, and throughout the acrosome reaction. As previously revealed in thin section electron microscopy, this fused membrane of the vacant acrosome continues to encircle the acrosomal process during the course of the sperm's approach to the egg surface (34). It is conceivable, therefore, that this heavy filipin labelling of the plasma membrane and of the outer acrosomal membrane in the periphery of the acrosomal opening may reflect a high sterol content which appears to confer stability on the membrane for preserving its structure throughout the process of fertilization.

On closer examination of the outer acrosomal membrane, the posterior region, in contrast to the apical region, remained sparsely distributed with filipin-sterol complexes during the acrosome reaction. It has been noted in certain membrane areas that membrane-associated proteins interfere with the formation of filipin-sterol complexes (8). In the present result, the sparsely distributed filipin-labelled area closely corresponds to the region where the outer acrosomal membrane is undercoated with an electron-dense material, as seen in the thin sections (28). Such sparse distribution of filipin labelling in the outer acrosomal membrane, therefore, could be caused by the interference of this electron-dense material with the filipin-sterol reaction by masking or linking the free sterols. This, in turn, may support membrane fluidity and facilitate the membrane folding which eventually occurs along the acrosomal process (34). However, the possibility of the latter being a result of a low sterol content in the membrane must also be considered.

As the inner acrosomal membrane is the innermost surface of the acrosome, it was difficult to obtain freeze-fracture replicas of it in the unreacted sperm. Thus, thin sections were used to visualize the filipin labelling, since membrane deformations produced by the filipin-sterol complexes were detectable in thin sections of the filipin-treated cells $(7,25,27)$. In the unreacted abalone sperm, filipin-induced deformation was seldom observed on the inner acrosomal membrane, which was densely packed with IMPs (29). Considering that this membrane tightly surrounds a bundle of actin filaments in the shaft region (36) and is undercoated with an electron-dense layer in the funnel-shaped region (28), our present result is in aggreement with those studies performed on other membrane systems, such as coated pits, desmosomes, 
tight junctions and intermediate junctions. These studies have reported that membrane domains with IMP aggregations or filamentous underlying usually lack filipin-sterol complexes $(7,14,20,26,32)$.

When rapid extension in the inner acrosomal membrane occurs to form the acrosomal process membrane, numerous densely packed IMPs found in the funnelshaped region of the inner acrosomal membrane scatter to form a patch-like pattern (29). In the filipin-treated sperm, identical alterations in the distribution of the IMPs were also observed. This suggests that the IMP distribution is not affected by filipin treatment. It is interesting that filipin labelling of the acrosomal process membrane appeared to show an inverse correlation with the distribution of IMPs; namely, the region where the IMPs are scattered from is replaced with filipin-sterol complexes as the acrosomal process elongates. Sterol is known to serve as a bulk modulator of fluidity in the membrane by interacting with phospholipids and, hence, affects the physical state of the membrane lipids $(6,23)$. Therefore, such characteristic filipin labelling could be interpreted as a reflection of the dynamic changes in the membrane which lead to the rapid formation of the acrosomal process membrane due to the effect of sterol on the membrane fluidity. Furthermore, since these lattice-like arrangements of IMPs were found to be twisted into a rhombic pattern, it may be presumed that the acrosomal process would elongate in a rotating motion or that following elongation, the acrosomal process would be rotated on its longitudinal axis.

In addition to regulating fluidity, sterol is considered to enhance the mechanical stability of the bilayer. After the elongation of the acrosomal process is completed, filipin-sterol complexes are more densely distributed in the anterior region of the acrosomal process membrane. This seems to give rigidity to the acrosomal process membrane. The results of this study may indicate that rapid regionalization of membrane sterols occurs in the membranes of the acrosomal region, which may be involved in modulating fluidity and stability of membranes which are requisite for the acrosome reaction.

Acknowledgements. The authors are grateful to Prof. T. Nagano and Dr. F. Suzuki for performing the freeze-fracture. The authors also wish to express their gratitude to the staff of Chiba Prefectural Fisheries Experimental Station for providing $H$. discus and for the use of their facilities.

This work was supported in part by a Grant-in-Aid for Scientific Research from the Ministry of Education, Science and Culture of Japan (No. 61740433).

\section{REFERENCES}

1. Bearer, E.L. and D.S. Friend. Modifications of anionic-lipid domains preceding membrane fusion in guinea pig sperm. J. Cell Biol. 92, 604-615, 1982

2. Bittman, R. Sterol-polyene antibiotic complexation: probe of membrane structure. Lipids 13, 686-691, 1978

3. De Kruijff, B., W.J. Gerritsen, A. Oerlemans, R.A. Demel and L.L.M. Van Deenen. Polyene antibiotic-sterol interactions in membranes of Acholeplasma laidlawii cells and lecithin liposomes. I. Specificity of the membrane permeability changes induced by the polyene antibiotics. Biochim. Biophys. Acta 339, 30-43, 1974

4. De Kruijff, B., W.J. Gerritsen, A. Oerlemans, P.W.M. Van Dijck, R.A. Demel and L.L.M. VAN DeEnen. Polyene antibiotic-sterol interactions in membranes of Acholeplasma laidlawii cells and lecithin liposomes. II. Temperature dependence of the polyene antibiotic-sterol complex formation. Biochim. Biophys. Acta 339, 44-56, 1974

5. De KruijfF, B. and R.A. Demel. Polyene antibiotic-sterol interactions in membranes of 
Acholeplasma laidlawii cells and lecithin liposomes. III. Molecular structure of the polyene antibiotic-cholesterol complexes. Biochim. Biophys. Acta 339, 57-70, 1974

6. Demel, R.A. and B. De Kruyff. The function of sterols in membranes. Biochim. Biophys. Acta 457, 109-132, 1976

7. Elias, P.M., D.S. Friend and J. Goerke. Membrane sterol heterogeneity. Freeze-fracture detection with saponins and filipin. J. Histochem. Cytochem. 27, 1247-1260, 1979

8. Feltkamp, C.A. and A.W.M. Van Der Waerden. Membrane-associated proteins affect the formation of filipin-cholesterol complexes in viral membranes. Exp. Cell Res. 140, 289-297, 1982

9. FLÉCHON, J.E. Sperm surface changes during the acrosome reaction as observed by freezefracture. Am. J. Anat. 174, 239-248, 1985

10. Friend, D.S., L. Orci, A. Perrelet and R. Yanagimachi. Membrane particle changes attending the acrosome reaction in guinea pig spermatozoa. J. Cell Biol. 74, 561-577, 1977

11. Friend, D.S. and P.M. Elias. Heterogeneity of filipin-sterol complexes in the guinea pig sperm plasma membrane. J. Cell Biol. 79, 216a, 1978

12. FrIEND, D.S. Freeze-fracture alterations in guinea pig sperm membranes preceding gamete fusion. in Membrane-Membrane Interactions, ed. Gilula N.B., Raven Press, New York, pp. $153-165,1980$

13. Friend, D.S. Plasma-membrane diversity in a highly polarized cell. J. Cell Biol. 93, 243-249, 1982

14. Gotow, T. and P.L. НаSнiмото. Filipin resistance in intermediate junction membranes of Guinea pig ependyma: possible relationship to filamentous underlying. J. Ultrastruct. Res. 84, $83-93,1983$

15. JAMIL, K., I.G. White and D.M. Dwarte. Calcium ionophore A23187 as a probe for freezefracture studies of membrane changes in the head of human spermatozoa. Arch. Andrology 8 , $1-9,1982$

16. KIKUCHI, S. and N. UKI. Technical study on artificial spawning of abalone, genus Haliotis. I. Relation between water temperature and advancing sexual maturity of Haliotis discus hannai Ino. Bull. Tohoku Reg. Fish. Res. Lab. 33, 69-78, 1974

17. KIKUCHI, S. and N. UKI. Technical study on artificial spawning of abalone, genus Haliotis. II. Effect of irradiated sea water with ultraviolet rays on inducing to spawn. Bull. Tohoku Reg. Fish. Res. Lab. 33, 79-86, 1974

18. Kitajima, Y., T. SekiYa and Y. Nozawa. Freeze-fracture ultrastructural alterations induced by filipin, pimaricin, nystatin and amphotericin B in the plasma membranes of Epidermophyton, Saccharomyces and red blood cells. A proposal of models for polyene-ergosterol complex-induced membrane lesions. Biochim. Biophys. Acta 445, 452-465, 1976

19. Kitajima, Y., T. SekiYA and Y. Nozawa. Sterol distribution in plasma membranes. Freezefracture cytochemistry by using a sterol-probe, filipin. Membrane 9, 184-201, 1984

20. Montesano, R., A. Perrelet, P. Vassalli and L. Orci. Absence of filipin-sterol complexes from large coated pits on the surface of culture cells. Proc. Natl. Acad. Sci. USA 76, 6391-6395, 1979

21. Montesano, R., P. Vassalli, A. Perrelet and L. Orci. Distribution of filipin-cholesterol complexes at sites of exocytosis. A freeze-fracture study of degranulating mast cells. Cell Biol. Int. Rep. 4, 975-984, 1980

22. Norman, A.W., R.A. Demel, B. De Kruijff, L.L.M. Van Deenen. Studies on the biological properties of polyene antibiotics. Evidence for the direct interaction of filipin with cholesterol. $J$. Biol. Chem. 247, 1918-1929, 1972

23. Oldfield, E. and D. Chapman. Dynamics of lipids in membranes: Heterogeneity and the role of cholesterol. FEBS Lett. 23, 285-297, 1972

24. Papahadjopoulos, D., M. Cowden and H. Kimelberg. Role of cholesterol in membranes. Effects on phospholipid-protein interactions, membrane permeability and enzymatic activity. Biochim. Biophys. Acta 330, 8-26, 1973

25. Pimenta, P.F.P. and W. De SouzA. Localization of filipin-sterol complexes in cell membranes of eosinophils. Histochemistry 80, 563-567, 1984

26. Robenek, H., W. Jung and R. Gebhardt. The topography of filipin-cholesterol complexes in the plasma membrane of cultured hepatocytes and their relation to cell junction formation. $J$. Ultrastruct. Res. 78, 95-106, 1982

27. Robinson, J.M. and M.J. Karnovsky. Evaluation of the polyene antibiotic filipin as a 
cytochemical probe for membrane cholesterol. J. Histochem. Cytochem. 28, 161-168, 1980

28. Sakai, Y.T., Y. Shiroy a and K. Haino-Fukushima. Fine structural changes in the acrosome reaction of the Japanese abalone, Haliotis discus. Dev. Growth Differ. 24, 531-542, 1982

29. SaKai, Y.T., F. SUZUKI and Y. Shiroya. Distribution of intramembrane particles and its changes during the acrosome reaction in spermatozoa of the Japanese abalone. Dev. Growth Differ. 27, 787-802, 1985

30. Sekiya, T., Y. Banno and Y. Nozawa. Reorganization of membrane cholesterol at an early stage of exocytosis (degranulation) in rat mast cells: Inferred from the distribution of filipin-cholesterol complexes. Cell Struct. Funct. 6, 301-312, 1981

31. Sekiya, T., T. Takenawa and Y. Nozawa. Reorganization of membrane cholesterol during membrane fusion in myogenesis in vitro: A study using the filipin-cholesterol complex. Cell Struct. Funct. 9, 143-155, 1984

32. Severs, N.J. and H. RobeneK. Detections of microdomains in biomembranes. An appraisal of recent developments in freeze-fracture cytochemistry. Biochim. Biophys. Acta 737, 373-408, 1983

33. Shinitzky, M. and P. Henkart. Fluidity of cell membranes. Current concepts and trends. Int. Rev. Cytol. 60, 121-147, 1980

34. Shiroya, Y. and Y.T. Sakai. Ultrastructural changes of the "Truncated Cone" during the acrosome reaction in Japanese abalone spermatozoa. Dev. Growth Differ. 26, 25-32, 1984

35. ShIRoYA, Y. and Y.T. SAKAI. Freeze-fracture study on acrosome reaction of abalone sperm with reference to membrane sterols. Zool. Sci. 3, 1041, 1986

36. Shiroya, Y., H. Hosoya, I. Mabuchi and Y.T. Sakai. Actin filament bundle in the acrosome of abalone spermatozoa. J. Exp. Zool. 239, 105-115, 1986

37. Tillack, T.W. and S.C. Kinsky. A freeze-etch study of the effects of filipin on liposomes and human erythrocyte membranes. Biochim. Biophys. Acta 323, 43-54, 1973

38. TOShImori, K., R. Higashi and C. ÖURA. Distribution of intramembranous particles and filipinsterol complexes in mouse sperm membranes: Polyene antibiotic filipin treatment. Am. J. Anat. 174, 455-470, 1985

39. Verkleij, A.J., B. De Kruijff, W.R. Gerritsen, R.A. Demel, L.L.M. Van Deenen and P.H.J. Ververgaert. Freeze-etch electron microscopy of erythrocytes, Acholeplasma laidlawii cells and liposomal membranes after the action of filipin and amphotericin B. Biochim. Biophys. Acta 291, $577-581,1973$

40. Yanagimachi, R. and F. Suzuki. A further study of lysolecithin-mediated acrosome reaction of guinea pig spermatozoa. Gamete Res. 11, 29-40, 1985 